\title{
Impact of Inferior Mesenteric Artery Lymph Node Metastasis on the Prognosis of Patients With Left- sided Colorectal Cancer: A Case Control Study
}

Kazuya Takabatake

Kyoto Prefectural University of Medicine

Tomohiro Arita ( $\nabla$ t-art@koto.kpu-m.ac.jp)

Division of Digestive Surgery, Department of Surgery, Kyoto Prefectural University of Medicine, Kyoto, 6028566, JAPAN

masayoshi Nakanishi

Kyoto Prefectural University of Medicine

Yoshiaki Kuriu

Kyoto Prefectural University of Medicine

Yasutoshi Murayama

Kyoto Prefectural University of Medicine

Hiroki Shimizu

Kyoto Prefectural University of Medicine

Michihiro Kudo

Kyoto Prefectural University of Medicine

Jun Kiuchi

Kyoto Prefectural University of Medicine

Toshiyuki Kosuga

Kyoto Prefectural University of Medicine

Hirotaka Konishi

Kyoto Prefectural University of Medicine

Ryo Morimura

Kyoto Prefectural University of Medicine

Atsushi Shiozaki

Kyoto Prefectural University of Medicine

Hisashi Ikoma

Kyoto Prefectural University of Medicine

Takeshi Kubota

Kyoto Prefectural University of Medicine

Hitoshi Fujiwara

Kyoto Furitsu Ika Daigaku Jibiinkoka Tokeibu Gekagaku Kyoshitsu 


\section{Kazuma Okamoto}

Kyoto Prefectural University of Medicine

Eigo Otsuji

Kyoto Prefectural University of Medicine

\section{Research article}

Keywords: Left-sided colorectal cancer, Inferior mesenteric artery, Lymph node metastasis

Posted Date: July 30th, 2020

DOI: https://doi.org/10.21203/rs.3.rs-41624/v1

License: (c) (1) This work is licensed under a Creative Commons Attribution 4.0 International License.

Read Full License 


\section{Abstract \\ Background:}

The clinical significance of metastasis in inferior mesenteric artery (IMA) lymph node in patients with leftsided colorectal cancer (LCRC) is unclear. The aim of this study was to investigate the impact of IMA lymph node metastasis (IMA-LN (+)) on the prognosis of patients with LCRC.

\section{Methods:}

A total of 292 patients with stage III LCRC and 111 patients with stage IV LCRC who underwent radical resection of the primary tumor between 2005 and 2016 were included. The clinicopathological features and prognosis, which were retrospectively obtained from medical records, were compared regarding IMALN (+).

\section{Results:}

IMA-LN (+) was observed in 10 patients with stage III LCRC (2.3\%). Moreover. $\geq 4$ metastatic lymph nodes $(p=0.001)$ and poorly differentiated type $(p=0.049)$ were more frequently observed in patients with IMALN (+) than in patients without IMA lymph node metastasis (IMA-LN (-)) in stage III; IMA-LN (+) patients had significantly worse overall survival (OS) than IMA-LN (-) patients in stage III $(p=0.015)$. Conversely, there was no significant difference between the OS of stage III IMA-LN (+) and stage IV patients $(p=$ 0.192). Likewise, there was no significant difference between the OS of stage III IMA-LN (+) and stage IV patients with distant metastatic lymph nodes only $(n=12)(p=0.294)$.

\section{Conclusion:}

The prognosis of IMA-LN (+) patients was worse than that of IMA-LN (-) patients in stage III LCRC; moreover, it was similar to that of patients with stage IV LCRC.

\section{Background}

Lymph node metastasis is one of the strongest prognostic factors in colorectal carcinoma. An increased number of cases of lymph node metastasis have been reported to be associated with poor prognosis in colorectal carcinoma [1, 2]; thus, according to the 8th edition of the TNM Classification of Malignant Tumors, the $\mathrm{N}$ stage is determined according to the number of metastatic lymph nodes [3]. Conversely, in the Japanese Classification of Colorectal, Appendiceal and Anal Carcinoma, the $\mathrm{N}$ stage is determined based on the main lymph node metastasis as well as the number of metastatic lymph nodes [4]. 
The inferior mesenteric artery (IMA) lymph nodes, which lie along the IMA from the origin of the IMA to the origin of the left colic artery, are defined as the regional lymph nodes and the main lymph nodes in left-sided colorectal cancer (LCRC) [3, 4]. A previous study has reported that stage III LCRC patients with IMA lymph node metastasis (IMA-LN (+)) had worse prognosis than those without it (IMA-LN (-)) [5]. Other studies have suggested that IMA-LN (+) can be an independent predictor of poor prognosis [6, 7]; thus, IMA lymph nodes could be included in not so much the regional lymph nodes as distant lymph nodes [6]. However, direct evidence is not provided, since there are no studies comparing the prognosis between stage III IMA-LN (+) and stage IV LCRC patients. Thus, the prognostic importance of IMA-LN (+) is still unclear.

In the present study, we aimed to evaluate the prognostic impact of IMA-LN (+) in stage III. Furthermore, we compared the prognosis of stage III IMA-LN (+) patients with that of stage IV LCRC patients.

\section{Methods}

\section{Patients and Data Collection}

Between 2005 and 2016, 1,566 consecutive patients underwent resection of the primary tumor for colorectal cancer at the Kyoto Prefectural University of Medicine. Among them, 403 patients with stage III or stage IV LCRC located in the descending colon, sigmoid colon, and rectum were involved in this study. The IMA lymph node was defined as the presence of lymph node from the origin of the inferior mesenteric artery to the origin of the left colic artery [4]. Patients were divided into three groups, according to the presence of IMA lymph node metastasis and the Union for International Cancer Control/American Joint Committee on Cancer Tumour, Node, Metastasis system version 8, and the Union for International Cancer Control (UICC). Moreover, stage IV patients were divided into two groups, according to the distant metastasis status.

Information about patients and their clinical course was collected from medical, operative, and pathology reports. Macroscopic and microscopic classifications of tumors were based on the UICC TNM staging system and the 8th edition of the Japanese Classification of Colorectal, Appendiceal, and Anal Carcinoma. Follow-up consisted of blood tests, gastrointestinal tract radiographies, endoscopic procedures, computed tomography, and ultrasonography. Follow-up procedures were performed every 3 months for at least 2 years and were continued periodically for up to 5 years. The median follow-up in 403 patients was 42 months.

Informed consent was obtained from all individual participants included in the study. All procedures performed in this study involving human participants were in accordance with the ethical standards of the institutional research committee and with the 1964 Helsinki declaration and its later amendments or comparable ethical standards. This study protocol was reviewed and approved by the Kyoto Prefectural University of Medicine Independent Ethics Committee (ERB-C-1178-1).

\section{Statistical Analysis}


Statistical analysis was performed using JMP version 14 (ASA Institute, Cary, NC, USA). Survival curves were calculated according to the Kaplan-Meier method. Differences between survival curves were examined by the Wilcoxon signed-rank test. Relationships between host and tumor factors were evaluated using Fisher's exact test of independence. In all analyses, $p<0.05$ values were considered significant.

\section{Results}

\section{In-Patient Setting}

Among 1566 patients undergoing resection of the primary tumor for colorectal cancer at our institution, 964 patients with stage $\mathbf{0}$, I, or II colorectal carcinoma, 187 patients with right-sided or transverse colon cancer, and 12 patients with other malignant diseases within 5 years were excluded. Eventually, 403 patients with stage III or stage IV LCRC located in the descending colon, sigmoid colon, and rectum were enrolled in this study. A total of 10 stage III LCRC patients had IMA lymph node metastasis (2.3\%), 282 patients had no IMA lymph node metastasis, and 111 patients were diagnosed with stage IV LCRC. Among 111 patients, 12 patients had distant lymph node metastasis only (Fig. 1).

\section{Tumor Factors in Patients with Stage III IMA-LN (+), IMA-LN $(-)$, or Stage IV LCRC}

First, tumor factors of patients in the three groups were investigated. Moreover, $\geq 4$ lymph nodes with metastasis $(p=0.001)$ and poorly differentiated type $(p=0.049)$ were more frequently observed in stage III IMA-LN (+) patients than in the IMA-LN (-) ones (Table 1). Conversely, no significant differences were observed between stage III IMA-LN (+) and stage IV LCRC patients in each tumor factor (Table 2).

\section{Long-Term Prognosis in Patients with Stage III IMA-LN (+), IMA-LN (-), and Stage IV LCRC}

Next, we also analyzed the long-term prognosis of the patients. The 5-year overall survival (OS) rates of stage III IMA-LN (+) and IMA-LN (-) patients were $57.1 \%$ and $78.1 \%$, respectively $(p=0.015)$ (Fig. $2 a)$. The 5 -year cancer-specific survival (CSS) rates were $66.7 \%$ and $83.6 \%$, respectively $(p=0.027$ ) (Fig. $2 b$ ). Conversely, the 5-year OS and CSS rates of stage IV LCRC patients were $24.8 \%$ and $26.1 \%$, respectively, and the prognosis of stage III IMA-LN (+) patients was not significantly different from stage IV LCRC patients (OS, $p=0.341$; CSS, $p=0.276$ ) (Fig. 2a, 2b).

Comparison of Prognosis and Tumor Factors in Patients with Stage III IM-LN(+) and Stage IV LCRC Patients with Distant Metastasis only

A total of 12 patients with distant lymph node metastasis only (stage IV (LYM)) were observed in stage IV LCRC. No significant differences were observed between tumor factors in stage III IMA-LN (+) and stage 
IV (LYM) LCRC (Table 3). In addition, the prognosis of stage IV (LYM) LCRC patients was not significantly different from that of stage III IMA-LN (+) patients (OS, $p=0.446$; CSS, $p=0.459)$ (Fig. 3a, 3b).

\section{Discussion}

In the present study, we have shown that stage III IMA-LN (+) patients had worse prognosis than stage III IMA-LN (-) patients. Conversely, no significant prognostic differences were observed between stage III IMA-LN (+) patients and stage IV LCRC patients, especially with distant lymph node metastasis only. These findings may indicate that IMA-LN (+) is not regarded as regional lymph node metastasis.

Our results regarding the poor prognosis of stage III IMA-LN (+) patients are consistent with those of previous studies.[6, 5, 7] Kang et al. have reported that the number of metastatic lymph nodes in stage III IMA-LN (+) patients was greater than in stage III IMA-LN (-) patients.[5] Thus, we compared the prognosis of IMA-LN (+) with IMA-LN (-) patients with $\geq 4$ metastatic lymph nodes, to exclude the influence of the number of metastatic lymph nodes. The prognosis of IMA-LN (+) tended to be inferior to that of IMA-LN $(-)$ (supplementary Fig. 1) and no great differences were observed in the tumor factors (supplementary Table 1), consistent with the previous report. Therefore, IMA-LN (+) may have a strong impact on prognosis regardless of the number of metastatic lymph nodes.

In the present study, the prognosis of stage III IMA-LN (+) patients was as poor as that of stage IV (LYM) LCRC patients. Newland et al. have reported that the apical lymph node involvement would implicate a spread to lymph node metastasis beyond the range of dissection [8]; Kang et al. have shown the high distant lymph node recurrence rate and significant association with para-aortic lymph node recurrence in stage III IMA-LN (+) patients [5]. Moreover, IMA-LN (+) was reported to be an independent prognostic factor in tumor factors including $\mathrm{T}, \mathrm{N}$, and $\mathrm{M}$ categories [6]. These findings, including our results, suggest that the IMA lymph nodes might be classified as systemic lymph nodes.

To improve the prognosis of IMA-LN (+) patients, the indications of postoperative adjuvant chemotherapy are important; for stage III patients who have undergone R0 resection, 5-FU-based chemotherapy is recommended [9]. Kim et al. have reported that adjuvant chemotherapy was an independent prognostic factor in IMA-LN (+) patients [6]. In our study, 7 among 10 stage III IMA-LN (+) patients received adjuvant chemotherapy. The 5-year OS rate of patients with adjuvant chemotherapy was $57.1 \%$ and that of those without adjuvant chemotherapy was $33.3 \%$. However, no significant difference was observed between the two groups (data not shown). The combination of 5-FU plus L-OHP is more effective for stage III CRC patients as adjuvant chemotherapy than mono-chemotherapy [10], whereas the combination of 5-FU plus CPT-11 or 5-FU plus molecular target drugs has not proven superior [11-14]; thus, currently, L-OHP combination chemotherapy may be more recommended for IMA-LN (+) patients, due to poor prognosis.

In the present study, seven IMA-LN (+) patients had sigmoid colon cancer, while three of them had rectal cancer. In general, descending/sigmoid colon and rectal cancers have different biological features because they have different lymphatic and blood vessels. However, neither significant prognostic nor recurrent rate difference was observed between the two groups (data not shown). Kang et al. have also 
reported that there was no significant difference in the systemic recurrence pattern between the sigmoid colon and rectal cancers [5]. These data may suggest that IMA lymph node metastasis is a significant prognostic factor, regardless of tumor location.

The present study has some limitations. It is a retrospective study in a single institute, with a small number of patients. In particular, the number of patients with IMA-LN (+) was small, because IMA-LN (+) is relatively rare $[6,15]$. However, regardless of the small number, our results may have meaningful suggestion on tumor stage classification that stage III IMA-LN (+) had worse prognosis than stage III IMALN (-), and there was no significant difference between stage III IMA-LN (+) and stage IV LCRC. Therefore, we wish to perform large size, prospective and randomized study.

\section{Conclusion}

In conclusion, the present study suggests that IMA-LN (+) can be a poor prognostic factor in stage III LCRC and cannot be regarded as an indicator of regional lymph node metastasis. Therefore, intensive adjuvant chemotherapy may be necessary to improve the poor prognosis in patients with stage III IMA-LN $(+)$.

\section{Abbreviations}

IMA, inferior mesenteric artery; LCRC, left-sided colorectal cancer; IMA-LN (+), the presence of inferior mesenteric artery lymph node metastasis; IMA-LN (-), the absence of inferior mesenteric artery lymph node metastasis; 5-FU, fluorouracil; L-OHP, oxaliplatin; CPT-11, irinotecan

\section{Declarations}

\section{Ethics approval and consent to participant}

All procedures performed in studies involving human participants were in accordance with the ethical standards of the institutional and/or national research committee and with the 1964 Helsinki Declaration and its later amendments or comparable ethical standards. This study protocol was reviewed and approved by the Kyoto Prefectural University of Medicine Independent Ethics Committee (ERB-C-11781).Written informed consent was obtained from all individual participants included in the study.

\section{Consent for publication}

Written consent for their personal or clinical details along with any identifying images to be published in this study was also obtained from all individual participants.

\section{Availability of data and materials}

The datasets and/or analyzed during the current study available fromthe corresponding author on reasonable request. 


\section{Competing interest}

The authors declare that they have no conflict of interest.

\section{Funding}

There is no funding supports for this paper.

\section{Authors' Contribution}

TA contributed to the study conception and design. Material preparation, data collection and analysis were performed by KT. The first draft of the manuscript was written by KT and all authors commented on previous versions of the manuscript. All authors approved the final manuscript and have agreed both to be personally accountable for the author's own contributions and to ensure that questions related to the accuracy or integrity of any partof the work are appropriately investigated, resolved, and the resolution documented in the literature.

\section{Acknowledgements}

We would like to thank Editage (www.editage.com) for English language editing.

\section{References}

1. Cohen AM, Tremiterra S, Candela F, Thaler HT, Sigurdson ER. Prognosis of node-positive colon cancer. Cancer. 1991;67:1859-61.

2. Le Voyer T, Sigurdson E, Hanlon A, Mayer R, Macdonald J, Catalano P, Haller D. Colon cancer survival is associated with increasing number of lymph nodes analyzed: a secondary survey of intergroup trial INT-0089. Journal of clinical oncology. 2003;21:2912-9.

3. Brierley JD, Gospodarowicz MK, Wittekind C. TNM classification of malignant tumours. New York: John Wiley \& Sons; 2016.

4. Japanese Society for Cancer of the Colon and Rectum. Japanese Classification of Colorectal Carcinoma. 8th edtion. (2013). Kanehara \& CoLtd, Japan.

5. Kang J, Hur H, Min BS, Kim NK, Lee KY. Prognostic impact of inferior mesenteric artery lymph node metastasis in colorectal cancer. Ann Surg Oncol. 2011. https://doi.org/10.1245/s10434-010-1291-x.

6. Kim JC, Lee KH, Yu CS, Kim HC, Kim JR, Chang HM, Kim JH, Kim JS, Kim TW. The clinicopathological significance of inferior mesenteric lymph node metastasis in colorectal cancer. Eur J Surg Oncol. 2004. https://doi.org/10.1016/j.ejso.2003.12.002.

7. Rao X, Zhang J, Liu T, Wu Y, Jiang Y, Wang P, Chen G, Pan Y, Wu T, Liu Y, Wan Y, Huang S, Wang X. Prognostic value of inferior mesenteric artery lymph node metastasis in cancer of the descending colon, sigmoid colon and rectum. Colorectal Dis. 2018. https://doi.org/10.1111/codi.14105. 
8. Newland RC, Dent OF, Lyttle MN, Chapuis PH, Bokey EL. Pathologic determinants of survival associated with colorectal cancer with lymph node metastases. A multivariate analysis of 579 patients. Cancer. 1994;73:2076-82.

9. Watanabe T, Muro K, Ajioka Y, Hashiguchi Y, et al. Japanese Society for Cancer of the Colon and Rectum (JSCCR) guidelines 2016 for the treatment of colorectal cancer. Int J Clin Oncol. 2018. https://doi.org/10.1007/s10147-017-1101-6.

10. Haller DG, Tabernero J, Maroun J, De Braud F, Price T, Van Cutsem E, Hill M, Gilberg F, Rittweger K, Schmoll H-J. Capecitabine plus oxaliplatin compared with fluorouracil and folinic acid as adjuvant therapy for stage III colon cancer. J Clin Oncol. 2011;29:1465-71.

11. Van Cutsem E, Labianca R, Bodoky G, Barone C, Aranda E, Nordlinger B, Topham C, Tabernero J, André T, Sobrero AF. Randomized phase III trial comparing biweekly infusional fluorouracil/leucovorin alone or with irinotecan in the adjuvant treatment of stage III colon cancer: PETACC-3. J Clin Oncol. 2009;27:3117-25.

12. de Gramont A, Van Cutsem E, Schmoll H-J, Tabernero J, Clarke S, Moore MJ, Cunningham D, Cartwright TH, Hecht JR, Rivera F. Bevacizumab plus oxaliplatin-based chemotherapy as adjuvant treatment for colon cancer (AVANT): a phase 3 randomised controlled trial. The lancet oncology. 2012;13:1225-33.

13. Taieb J, Tabernero J, Mini E, Subtil F, Folprecht G, Van Laethem J-L, Thaler J, Bridgewater J, Petersen $\mathrm{LN}$, Blons $\mathrm{H}$. Oxaliplatin, fluorouracil, and leucovorin with or without cetuximab in patients with resected stage III colon cancer (PETACC-8): an open-label, randomised phase 3 trial. The Lancet Oncology. 2014;15:862-73.

14. Kerr RS, Love S, Segelov E, Johnstone E, Falcon B, Hewett P, Weaver A, Church D, Scudder C, Pearson $S$. Adjuvant capecitabine plus bevacizumab versus capecitabine alone in patients with colorectal cancer (QUASAR 2): an open-label, randomised phase 3 trial. The Lancet Oncology. 2016;17:154357.

15. Kanemitsu Y, Hirai T, Komori K, Kato T. Survival benefit of high ligation of the inferior mesenteric artery in sigmoid colon or rectal cancer surgery. British Journal of Surgery: Incorporating European Journal of Surgery Swiss Surgery. 2006;93:609-15.

\section{Tables}

Table 1.

Tumor factors of stage III IMA-LN (-) and stage III IMA-LN (+) patients 


\begin{tabular}{|c|c|c|c|c|}
\hline \multirow[t]{2}{*}{ Factors } & \multirow[t]{2}{*}{ Group } & \multirow{2}{*}{$\begin{array}{l}\text { IMA-LN (-) } \\
\mathrm{n}=\mathbf{2 8 2}\end{array}$} & \multirow{2}{*}{$\begin{array}{l}\text { IMA-LN (+) } \\
\mathrm{n}=10\end{array}$} & \multirow[t]{2}{*}{$P$ value } \\
\hline & & & & \\
\hline \multirow[t]{2}{*}{ Age (years) } & $<66$ & $141(50 \%)$ & $4(40 \%)$ & 0.75 \\
\hline & $\geq 66$ & $141(50 \%)$ & $6(60 \%)$ & \\
\hline \multirow[t]{2}{*}{ Sex } & Female & 137 (49\%) & $5(50 \%)$ & 0.535 \\
\hline & Male & $145(51 \%)$ & $5(50 \%)$ & \\
\hline \multirow[t]{2}{*}{ Operation time (min) } & $<240$ & $132(47 \%)$ & $4(40 \%)$ & 0.758 \\
\hline & $\geq 240$ & $152(53 \%)$ & $6(60 \%)$ & \\
\hline \multirow[t]{2}{*}{ Bleeding (ml) } & $<45$ & 148 (52\%) & 7 (70\%) & 1.000 \\
\hline & $\geq 45$ & $134(48 \%)$ & $3(30 \%)$ & \\
\hline \multirow[t]{3}{*}{ Tumor location } & Descending & $9(3 \%)$ & $0(0 \%)$ & 0.142 \\
\hline & Sigmoid & $107(38 \%)$ & $7(70 \%)$ & \\
\hline & Rectum & $166(59 \%)$ & $3(30 \%)$ & \\
\hline \multirow[t]{2}{*}{ T factor ${ }^{a}, *$} & $\mathrm{~T} 1,2$ & $68(24 \%)$ & $0(0 \%)$ & 0.124 \\
\hline & $\mathrm{T} 3,4$ & $214(76 \%)$ & $10(100 \%)$ & \\
\hline \multirow[t]{2}{*}{ Number of lymph node metastasis * } & $<4$ & $221(78 \%)$ & $3(30 \%)$ & 0.001 \\
\hline & $\geq 4$ & $61(22 \%)$ & $7(70 \%)$ & \\
\hline \multirow[t]{2}{*}{ Histopathological type ${ }^{\mathrm{b},} \dagger$} & Poorly differentiated & $23(8 \%)$ & $3(30 \%)$ & 0.049 \\
\hline & Well differentiated & $259(92 \%)$ & $7(70 \%)$ & \\
\hline \multirow[t]{2}{*}{ Tumor size (mm) } & $<45$ & $162(57 \%)$ & $4(40 \%)$ & 0.203 \\
\hline & $\geq 45$ & $110(43 \%)$ & $6(60 \%)$ & \\
\hline \multirow[t]{2}{*}{ Venous invasion $†$} & Absent & $127(45 \%)$ & $4(40 \%)$ & 1.000 \\
\hline & Present & $155(55 \%)$ & $6(60 \%)$ & \\
\hline \multirow[t]{2}{*}{ Lymphatic invasion $†$} & Absent & $108(38 \%)$ & $1(10 \%)$ & 0.096 \\
\hline & Present & $174(62 \%)$ & $9(90 \%)$ & \\
\hline \multirow[t]{2}{*}{ CA19-9 (U/ml) } & $<37$ & $244(87 \%)$ & $7(70 \%)$ & 0.152 \\
\hline & $\geq 37$ & $35(12 \%)$ & $3(30 \%)$ & \\
\hline \multirow[t]{2}{*}{ CEA (ng/ml) } & $<5$ & 167 (59\%) & $5(50 \%)$ & 0.745 \\
\hline & $\geq 5$ & $113(40 \%)$ & $5(50 \%)$ & \\
\hline
\end{tabular}


IMA-LN (-), the absence of the inferior mesenteric artery lymph node metastasis

IMA-LN (+), the presence of the inferior mesenteric artery lymph node metastasis

CA19-9, carbohydrate antigen 19-9; CEA, carcinoembryonic antigen

a, Depth of invasion: T1, tumor invasion of the lamina propria or submucosa; T2, tumor invasion of the muscularis propria; $\mathrm{T} 3$, tumor invasion of the sub serosa or within adventitia; $\mathrm{T} 4$, tumor penetration of the serosa or tumor invasion of adjacent organs

b, Histological type: well differentiated, well or moderately differentiated adenocarcinoma; poorly differentiated, poorly differentiated adenocarcinoma, signet ring cell carcinoma or mucinous carcinoma *, According to the Union for International Cancer Control/American Joint Committee on Cancer Tumour, Node, Metastasis system version 8 , and the Union for International Cancer Control

†, According to 8th edition of the Japanese Classification of Colorectal, Appendiceal, and Anal Carcinoma

Table 2.

Tumor factors of stage III IMA-LN (+) and stage IV LCRC patients 


\begin{tabular}{|c|c|c|c|c|}
\hline Factors & Group & $\begin{array}{l}\text { IMA-LN (+) } \\
n=10\end{array}$ & $\begin{array}{l}\text { Stage IV } \\
n=111\end{array}$ & $P$ value \\
\hline \multirow[t]{2}{*}{ Age (years) } & $<66$ & $4(40 \%)$ & $52(47 \%)$ & 0.751 \\
\hline & $\geq 66$ & $6(60 \%)$ & $59(53 \%)$ & \\
\hline \multirow[t]{2}{*}{ Sex } & Female & $5(50 \%)$ & 49 (44\%) & 0.751 \\
\hline & Male & $5(50 \%)$ & $62(56 \%)$ & \\
\hline \multirow[t]{2}{*}{ Operation time (min) } & $<240$ & $4(40 \%)$ & $51(46 \%)$ & 0.754 \\
\hline & $\geq 240$ & $6(60 \%)$ & $60(54 \%)$ & \\
\hline \multirow[t]{2}{*}{ Bleeding (ml) } & $<45$ & 7 (70\%) & 48 (43\%) & 0.747 \\
\hline & $\geq 45$ & $3(30 \%)$ & $63(57 \%)$ & \\
\hline \multirow[t]{3}{*}{ Tumor location } & Descending & $0(0 \%)$ & $7(6 \%)$ & 0.154 \\
\hline & Sigmoid & $7(70 \%)$ & $40(36 \%)$ & \\
\hline & Rectum & $3(30 \%)$ & $64(58 \%)$ & \\
\hline \multirow[t]{2}{*}{ T factor ${ }^{a}, *$} & $\mathrm{~T} 1,2$ & $0(0 \%)$ & $3(2 \%)$ & 1.000 \\
\hline & $\mathrm{T} 3,4$ & $10(100 \%)$ & $108(98 \%)$ & \\
\hline \multirow[t]{2}{*}{ Number of lymph node metastasis * } & $<4$ & $3(30 \%)$ & $63(57 \%)$ & 0.183 \\
\hline & $\geq 4$ & $7(70 \%)$ & $48(43 \%)$ & \\
\hline \multirow[t]{2}{*}{ Histopathological type ${ }^{\mathrm{b},} \dagger$} & Poorly differentiated & $3(30 \%)$ & $17(15 \%)$ & 0.366 \\
\hline & Well differentiated & $7(70 \%)$ & 94 (85\%) & \\
\hline \multirow[t]{2}{*}{ Tumor size (mm) } & $<45$ & $4(40 \%)$ & $28(25 \%)$ & 0.453 \\
\hline & $\geq 45$ & $6(60 \%)$ & $83(75 \%)$ & \\
\hline \multirow[t]{2}{*}{ Venous invasion $†$} & Absent & $4(40 \%)$ & $23(21 \%)$ & 0.228 \\
\hline & Present & $6(60 \%)$ & $88(79 \%)$ & \\
\hline \multirow[t]{2}{*}{ Lymphatic invasion $†$} & Absent & $1(10 \%)$ & $36(32 \%)$ & 0.280 \\
\hline & Present & $9(90 \%)$ & 75 (68\%) & \\
\hline \multirow[t]{2}{*}{ CA19-9 (U/ml) } & $<37$ & $7(70 \%)$ & $60(54 \%)$ & 0.510 \\
\hline & $\geq 37$ & $3(30 \%)$ & $50(45 \%)$ & \\
\hline \multirow[t]{2}{*}{ CEA (ng/ml) } & $<5$ & $5(50 \%)$ & $25(23 \%)$ & 0.118 \\
\hline & $\geq 5$ & $5(50 \%)$ & 85 (76\%) & \\
\hline
\end{tabular}


IMA-LN (+), the presence of the inferior mesenteric artery lymph node metastasis

LCRC, left-sided colorectal carcinoma

CA19-9, carbohydrate antigen 19-9; CEA, carcinoembryonic antigen

a, Depth of invasion: T1, tumor invasion of the lamina propria or submucosa; $T 2$, tumor invasion of the muscularis propria; $\mathrm{T} 3$, tumor invasion of the sub serosa or within adventitia; $\mathrm{T} 4$, tumor penetration of the serosa or tumor invasion of adjacent organs

b, Histological type: well differentiated, well or moderately differentiated adenocarcinoma; poorly differentiated, poorly differentiated adenocarcinoma, signet ring cell carcinoma or mucinous carcinoma *, According to the Union for International Cancer Control/American Joint Committee on Cancer Tumour, Node, Metastasis system version 8 , and the Union for International Cancer Control

${ }^{\dagger}$, According to 8th edition of the Japanese Classification of Colorectal, Appendiceal, and Anal Carcinoma

Table 3

Tumor factors of stage III IMA-LN (+) and stage IV (LYM) LCRC patients 


\begin{tabular}{|c|c|c|c|c|}
\hline \multirow[t]{2}{*}{ Factors } & \multirow[t]{2}{*}{ Group } & \multirow{2}{*}{$\begin{array}{l}\underset{(+)}{\operatorname{IMA} A-L N} \\
n=10\end{array}$} & \multirow{2}{*}{$\begin{array}{l}\text { Stage IV } \\
\text { (LYM) } \\
n=12\end{array}$} & \multirow[t]{2}{*}{$P$ value } \\
\hline & & & & \\
\hline \multirow[t]{2}{*}{ Age (years) } & $<66$ & $4(40 \%)$ & $8(67 \%)$ & 0.391 \\
\hline & $\geq 66$ & $6(60 \%)$ & $4(33 \%)$ & \\
\hline \multirow[t]{2}{*}{ Sex } & Female & $5(50 \%)$ & $6(50 \%)$ & 1.000 \\
\hline & Male & $5(50 \%)$ & $6(50 \%)$ & \\
\hline \multirow[t]{2}{*}{ Operation time (min) } & $<240$ & $4(40 \%)$ & $3(25 \%)$ & 0.652 \\
\hline & $\geq 240$ & $6(60 \%)$ & $9(75 \%)$ & \\
\hline \multirow[t]{2}{*}{ Bleeding (ml) } & $<45$ & $7(70 \%)$ & $1(8 \%)$ & 0.055 \\
\hline & $\geq 45$ & $3(30 \%)$ & $11(92 \%)$ & \\
\hline \multirow[t]{3}{*}{ Tumor location } & Descending & $0(0 \%)$ & $1(8 \%)$ & 0.198 \\
\hline & Sigmoid & $7(70 \%)$ & $4(33 \%)$ & \\
\hline & Rectum & $3(30 \%)$ & $7(59 \%)$ & \\
\hline \multirow[t]{2}{*}{ T factor ${ }^{a, *}$} & $\mathrm{~T} 1,2$ & $0(0 \%)$ & $0(0 \%)$ & NA \\
\hline & $\mathrm{T} 3,4$ & $10(100 \%)$ & $12(100 \%)$ & \\
\hline \multirow{2}{*}{$\begin{array}{l}\text { Number of lymph node metastasis } \\
\star\end{array}$} & $<4$ & $3(30 \%)$ & $3(25 \%)$ & 1.000 \\
\hline & $\geq 4$ & $7(70 \%)$ & $9(75 \%)$ & \\
\hline \multirow[t]{2}{*}{ Histopathological type ${ }^{\mathrm{b},}+$} & $\begin{array}{l}\text { Poorly } \\
\text { differentiated }\end{array}$ & $3(30 \%)$ & $6(50 \%)$ & 0.415 \\
\hline & Well differentiated & $7(70 \%)$ & $6(50 \%)$ & \\
\hline \multirow[t]{2}{*}{ Tumor size (mm) } & $<45$ & $4(40 \%)$ & $3(25 \%)$ & 0.652 \\
\hline & $\geq 45$ & $6(60 \%)$ & $9(75 \%)$ & \\
\hline \multirow[t]{2}{*}{ Venous invasion $†$} & Absent & $4(40 \%)$ & $3(25 \%)$ & 0.652 \\
\hline & Present & $6(60 \%)$ & $9(75 \%)$ & \\
\hline \multirow[t]{2}{*}{ Lymphatic invasion $†$} & Absent & $1(10 \%)$ & $2(17 \%)$ & 1.000 \\
\hline & Present & $9(90 \%)$ & $10(83 \%)$ & \\
\hline \multirow[t]{2}{*}{ CA19-9 (U/ml) } & $<37$ & $7(70 \%)$ & $7(58 \%)$ & 0.675 \\
\hline & $\geq 37$ & $3(30 \%)$ & $5(42 \%)$ & \\
\hline CEA (ng/ml) & $<5$ & $5(50 \%)$ & $2(17 \%)$ & 0.172 \\
\hline
\end{tabular}


IMA-LN (+), the presence of the inferior mesenteric artery lymph node metastasis

Stage IV (LYM), stage IV only with distant lymph node metastasis

LCRC, left-sided colorectal carcinoma

CA19-9, carbohydrate antigen 19-9; CEA, carcinoembryonic antigen

a, Depth of invasion: $\mathrm{T} 1$, tumor invasion of the lamina propria or submucosa; $T 2$, tumor invasion of the muscularis propria; $\mathrm{T} 3$, tumor invasion of the sub serosa or within adventitia; $\mathrm{T} 4$, tumor penetration of the serosa or tumor invasion of adjacent organs

b, Histological type: well differentiated, well or moderately differentiated adenocarcinoma; poorly differentiated, poorly differentiated adenocarcinoma, signet ring cell carcinoma or mucinous carcinoma

*, According to the Union for International Cancer Control/American Joint Committee on Cancer Tumour, Node, Metastasis system version 8 , and the Union for International Cancer Control

${ }^{\dagger}$, According to 8th edition of the Japanese Classification of Colorectal, Appendiceal, and Anal Carcinoma

\section{Figures}




\begin{tabular}{|l|l|}
1566 consecutive patients underwent curative resection or \\
resection of the primary tumor for colorectal cancer \\
between 2005 and 2016.
\end{tabular}

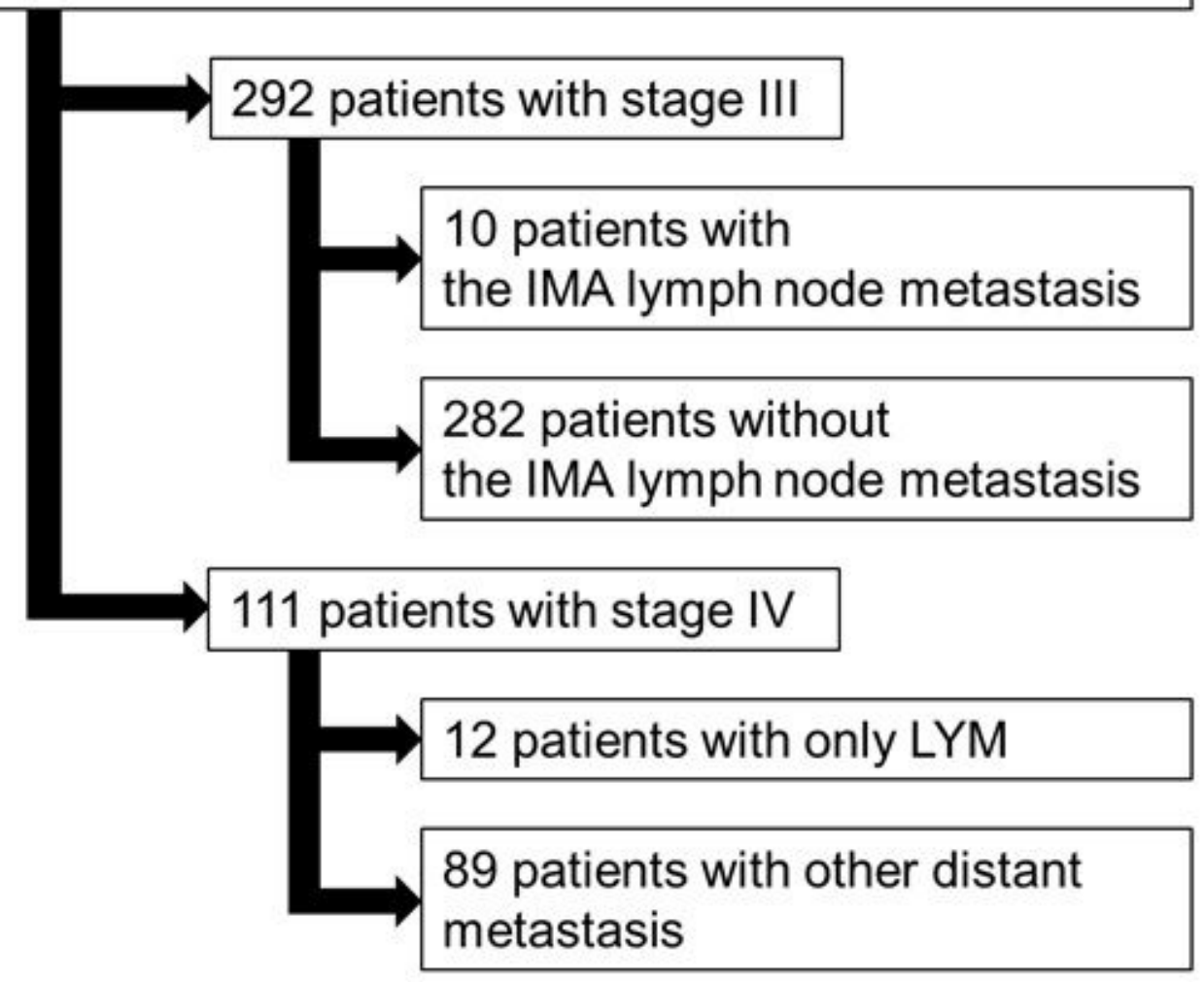

Figure 1

A total of 403 patients with stage III or stage IV left-sided colorectal cancer, whounderwent curative resection or resection of the primary tumor were enrolled in this study. The patients were grouped according to the TNM staging system and lymph node metastasis status of the IMA. IMA:inferior mesenteric artery LYM: distant lymph node metastasis 
Fig. 2.

a

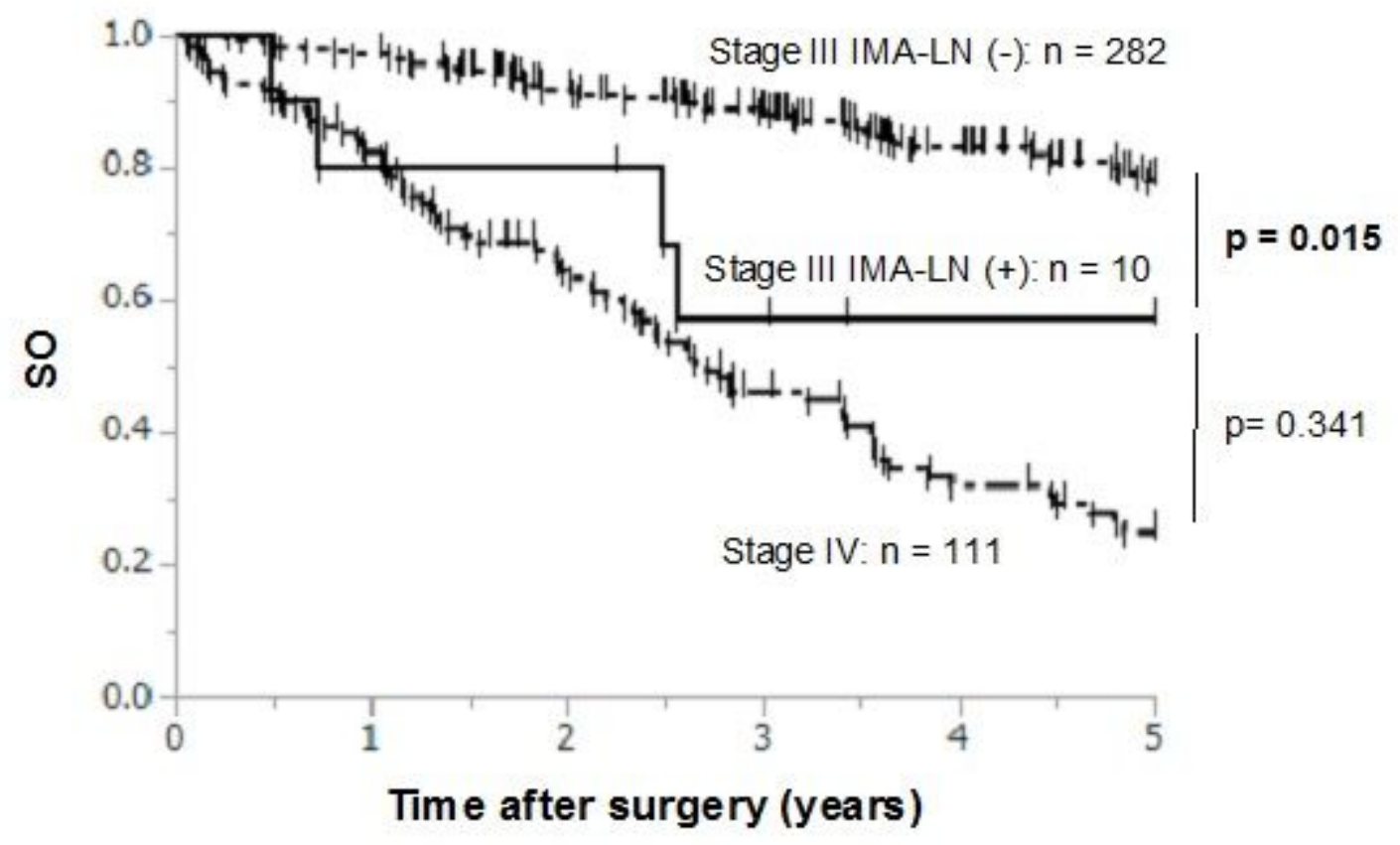

b

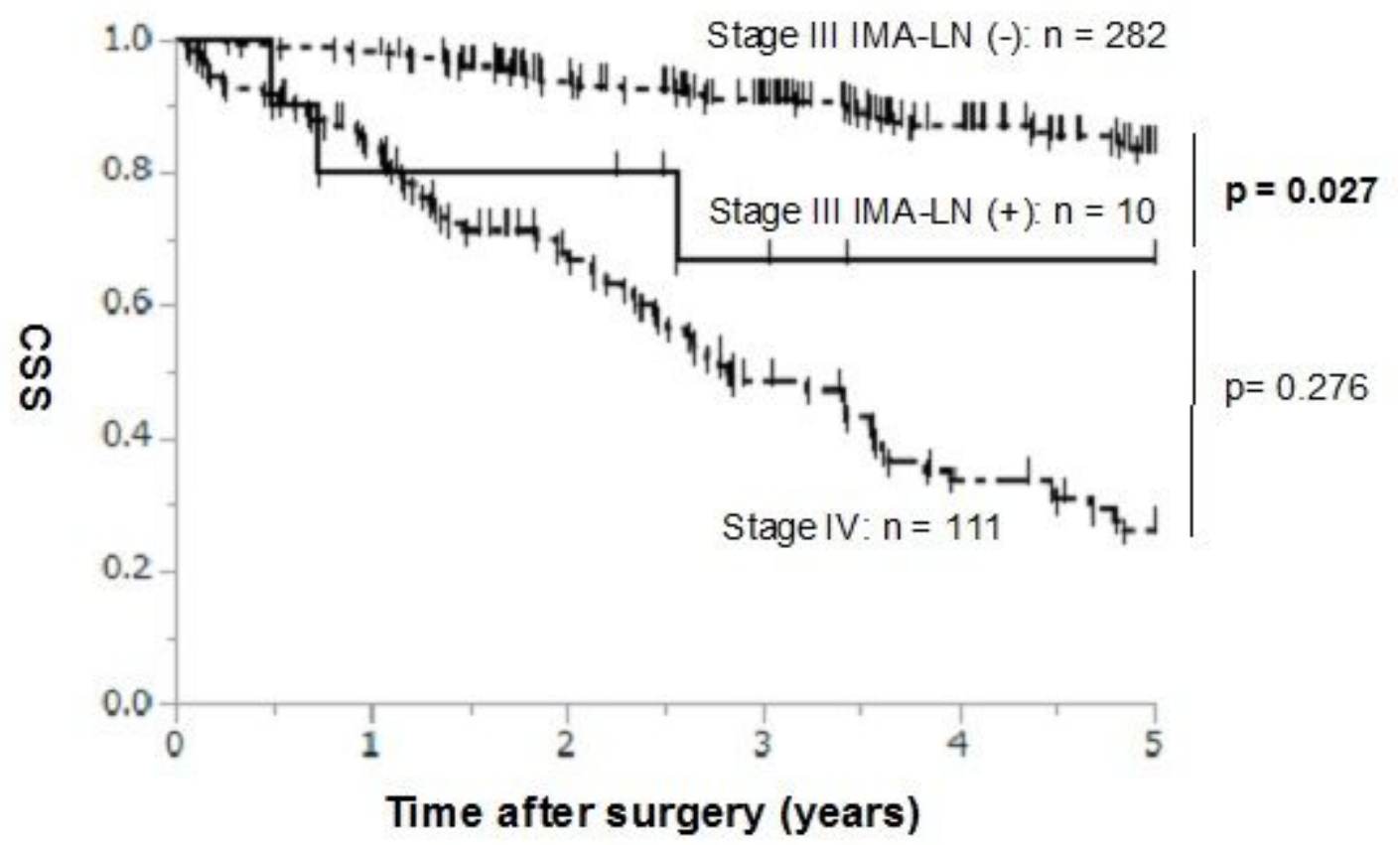

Figure 2

(a) Overall survival (OS) and (b) cancer-specific survival (CSS), based on the stage and the status of the IMA lymph node metastasis. IMA-LN (-):absence of the IMA mesenteric lymph node metastasis IMA-LN $(+)$ :presence of the IMA mesenteric lymph node metastasis 
Fig. 3.

a

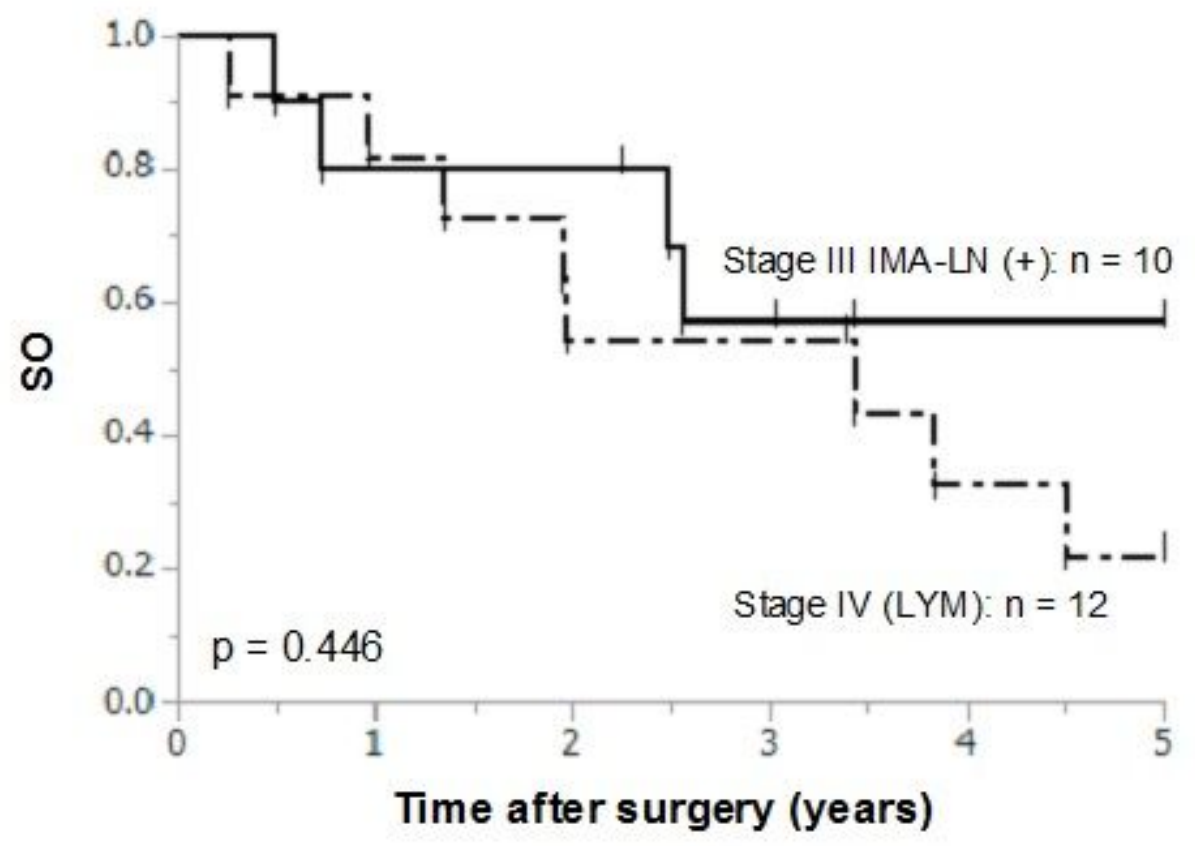

b

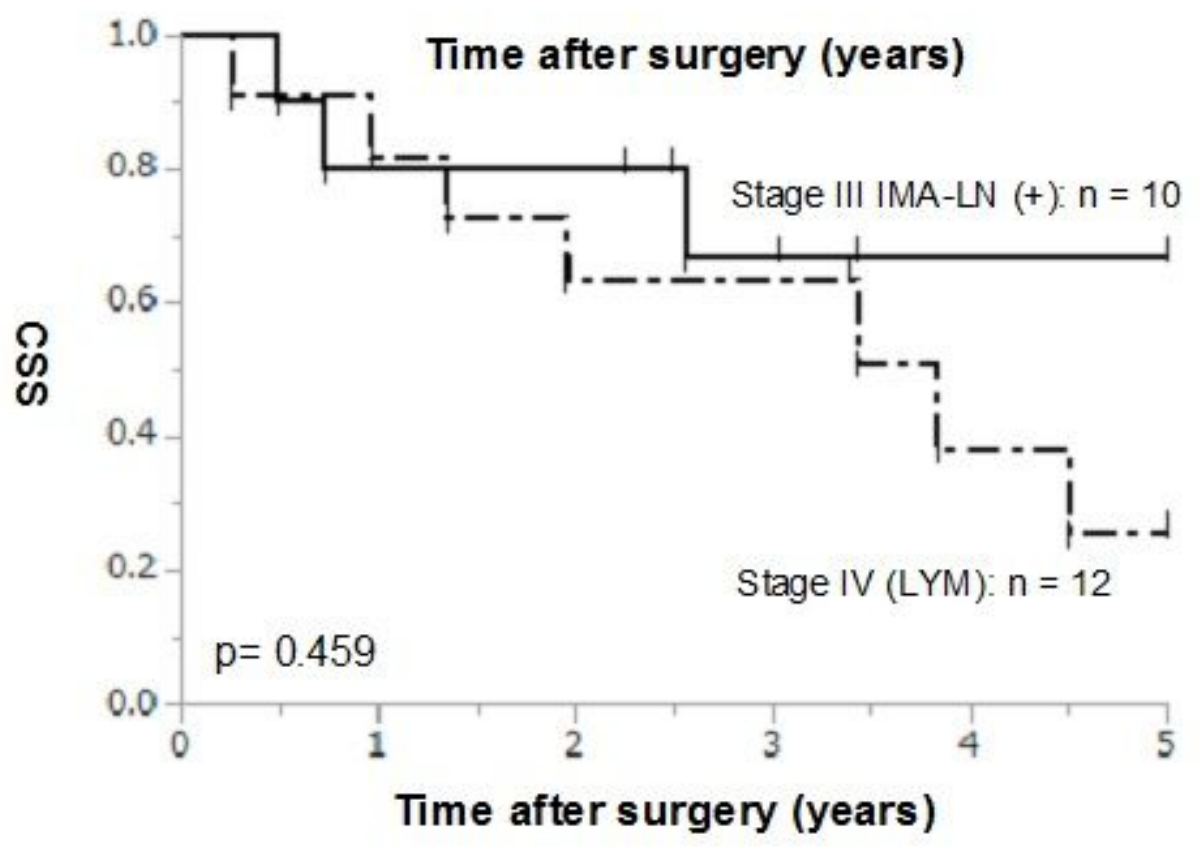

Figure 3

(a) Overall survival (OS) and (b) cancer specific survival (CSS), compared between stage III IMA-LN (+) and stage IV (LYM) LCRC patients. IMA-LN (+): presence of the IMA mesenteric lymph node metastasis LCRC: left-sided colorectal cancer. Stage IV (LYM), stage IV left-sided colorectal cancer with distant lymph node metastasis only. 
This is a list of supplementary files associated with this preprint. Click to download.

- ImpactofIMALNmetastasisonprognosisstable.docx

- ImpactofIMALNmetastasisonprognosissFig.docx.docx 